\title{
Continental-scale genomic analysis suggests shared post-admixture adaptation in the Americas
}

\author{
Linda Ongaro ${ }^{1, *}, \dagger$, Mayukh Mondal ${ }^{1}$, Rodrigo Flores ${ }^{1}$, Davide Marnetto ${ }^{1}$, \\ Ludovica Molinaro ${ }^{1}$, Marta E. Alarcón-Riquelme ${ }^{2}$, Andrés Moreno-Estrada ${ }^{3}$, \\ Nedio Mabunda ${ }^{4}$, Mario Ventura ${ }^{5}$, Kristiina Tambets ${ }^{1}$, Garrett Hellenthal ${ }^{6, \ddagger}$, \\ Cristian Capelli ${ }^{7,8}$, Toomas Kivisild ${ }^{9}$, Mait Metspalu ${ }^{1}$, Luca Pagani ${ }^{1,10, \dagger}$ and \\ Francesco Montinaro ${ }^{1,5,9}$
}

${ }^{1}$ Estonian Biocentre, Institute of Genomics, University of Tartu, Riia 23b, Tartu 51010, Estonia, ${ }^{2}$ Department of Medical Genomics, GENYO. Centro Pfizer - Universidad de Granada - Junta de Andalucía de Genómica e Investigación Oncológica, Av de la Ilustración 114, Parque Tecnológico de la Salud (PTS), Granada 18016, Spain, ${ }^{3}$ National Laboratory of Genomics for biodiversity (LANGEBIO), CINVESTAV, Irapuato, Guanajuato 36821, Mexico, ${ }^{4}$ Instituto Nacional de Saúde, Distrito de Marracuene, Estrada Nacional No. 1, Província de Maputo, Maputo 1120, Mozambique, ${ }^{5}$ Department of Biology-Genetics, University of Bari, Bari 70126, Italy, ${ }^{6}$ Department of Genetics, Evolution and Environment and UCL Genetics Institute, University College London, London WC1E 6BT, UK, ${ }^{7}$ Department of Zoology, University of Oxford, 11a Mansfield Road, OX1 3SZ, Oxford, UK, ${ }^{8}$ Department of Chemistry, Life Sciences and Environmental Sustainability, University of Parma, Parco Area delle Scienze 11/a, 43124, Parma, Italy, ${ }^{9}$ Department of Human Genetics, KU Leuven, Herestraat 49 - box 602, Leuven B-3000, Belgium and ${ }^{10}$ Department of Biology, University of Padua, Via U. Bassi 58/B, 35131, Padua, Italy

*To whom correspondence should be addressed at: Estonian Biocentre, Institute of Genomics, University of Tartu, Tartu, Riia 23b, 51010, Estonia. Email: linda.ongaro@ut.ee.

\begin{abstract}
American populations are one of the most interesting examples of recently admixed groups, where ancestral components from three major continental human groups (Africans, Eurasians and Native Americans) have admixed within the last 15 generations. Recently, several genetic surveys focusing on thousands of individuals shed light on the geography, chronology and relevance of these events. However, even though gene flow could drive adaptive evolution, it is unclear whether and how natural selection acted on the resulting genetic variation in the Americas. In this study, we analysed the patterns of local ancestry of genomic fragments in genome-wide data for $\sim 6000$ admixed individuals from 10 American countries. In doing so, we identified regions characterized by a divergent ancestry profile (DAP), in which a significant over or under ancestral representation is evident. Our results highlighted a series of genomic regions with DAPs associated with immune system response and relevant medical traits, with the longest DAP region encompassing the human leukocyte antigen locus. Furthermore, we found that DAP regions are enriched in genes linked to cancer-related traits and autoimmune diseases. Then, analysing the biological impact of these regions, we showed that natural selection could have acted
\end{abstract}

${ }^{\dagger}$ Linda Ongaro, http://orcid.org/0000-0003-1900-3394

‡Garrett Hellenthal, http://orcid.org/0000-0002-5760-8020

"These authors contributed equally as senior authors.

Received: January 18, 2021. Revised: June 21, 2021. Accepted: June 23, 2021

(C) The Author(s) 2021. Published by Oxford University Press.

This is an Open Access article distributed under the terms of the Creative Commons Attribution Non-Commercial License

(http://creativecommons.org/licenses/by-nc/4.0/), which permits non-commercial re-use, distribution, and reproduction in any medium,

provided the original work is properly cited. For commercial re-use, please contact journals.permissions@oup.com 
preferentially towards variants located in coding and non-coding transcripts and characterized by a high deleteriousness score. Taken together, our analyses suggest that shared patterns of post admixture adaptation occurred at a continental scale in the Americas, affecting more often functional and impactful genomic variants.

\section{Introduction}

The genomic variation of a substantial proportion of the individuals living in the Americas is the result of admixture involving Native American, European and African populations, together with minor recent contributions from Asia, as the results of deportation and mass migrations followed by admixture episodes (1-4).

Although many studies uncovered the complexity of the admixture dynamics in the continents (1,3-5), addressing the role of adaptive introgression in shaping the modern-day variation of American populations has been particularly challenging. In fact, the high variance in individual continental ancestries, due to very recent and still ongoing admixture, makes it hard to apply classical natural selection tools based on the distribution of genetic variation along the genome (6-9). Following the advent of high-throughput sequencing and the development of statistical tools aimed at inferring the ancestry of specific genomic regions, a commonly used approach to tackle this question focuses on loci showing ancestral proportions across the entire population that significantly diverge from wholegenome estimates (10). Regions enriched or depleted for a given ancestry are usually interpreted as targets of natural selection, driven by post admixture adaptive pressures. Despite the large number of studies harnessing these methods, the results have not been consistent and were often not replicated. For example, the analysis of $\sim 2000$ African Americans found putatively enriched loci (deviating more than three standard deviations) for European and African ancestries (11). However, a subsequent study analysing $\sim 29000$ individuals and applying a genomewide significance threshold did not find any statistically significant diverging loci, highlighting the possibility of false-positive detection when less conservative thresholds are applied (12).

Furthermore, most of the selection scans performed so far focused either on single populations or multiple groups of small sample sizes, increasing the chance of collecting false positives.

Nevertheless, some investigations of local ancestry tracts distributions in individuals from Peru, Puerto Rico, Mexico and Colombia are concordant in suggesting rapid natural selection in genomic loci associated with immune response, such as the major histocompatibility complex (MHC) (13-18).

In this study, we analyse local ancestry tracts distribution for 5828 American individuals from 19 populations to elucidate the role of post-admixture selection in shaping the genetic variation of the Americas.

In doing so, we focused only on signals shared across multiple populations, reducing the inference of false positives and at the same time highlighting shared or convergent episodes of selection as proposed in Yelmen et al. (19). Moreover, given the Columbian exchange phenomenon, the asymmetric resistance to pathogens such as Measles and Smallpox in Europeans and not in Americans, and Syphilis in Americans but not in Europeans, we specifically looked for signals in genes associated with the biology of these diseases (20-22).

Our results highlighted a series of genomic regions with divergent ancestry profiles (DAPs) associated with relevant medical traits such as the MHC, and others with recurring association to cholesterol and triglyceride levels, systemic lupus erythematosus and blood protein levels. Furthermore, we found that the single nucleotide polymorphisms (SNPs) belonging to DAP windows are enriched in genes linked to cancer-related traits and autoimmune diseases. Lastly, the analysis of the functional impact and annotation of the DAP windows revealed a more prominent role of natural selection on variants located in coding and non-coding transcripts and characterized by a stronger annotated impact.

\section{Results}

\section{Evaluation of signals identified in multiple populations}

We aimed to identify genomic regions showing significant deviations in local ancestry assignment from the average ancestry proportion in a given population. We applied the Local Ancestry RFMix software (23) on data from the Americas (Table 1) as presented in Ongaro et al. (1), in which the ancestry composition of the admixed individuals was deconvoluted using four putative source groups (Africa: 190 individuals; Europe: 289 individuals; Americas: 67 individuals; Asia: 213 individuals; Supplementary Material, Table S1A). We have here excluded the latter, given the low proportion of Asian ancestry detected in our previous analysis (1).

We evaluated the local ancestry output using two differently assembled datasets, as described in the Materials and Methods section. From this point on, we performed all the analyses for each ancestry separately. Briefly, to take into account possible errors when populations or individuals have extreme ancestry proportions, for the first dataset (20Pop dataset), we focused only on populations with $>20 \%$ of a given ancestry; while for the second one (1090Ind dataset), we excluded individuals characterized by extreme proportions of any given ancestry $(<10 \%$ and $>90 \%$ ).

We estimated the ancestry specific Z-score for each SNP (data available at https://doi.org/10.5281/zenodo.4446628), as explained in the Materials and Methods section.

We aggregated the SNP-based output in $100 \mathrm{~kb}$ nonoverlapping windows, retaining only windows containing $>5$ SNPs, and annotated as DAP those with at least one SNP with a significant $\mathrm{Z}$ score $(>|3|)$. We selected only DAP signals replicated in at least two populations to reduce the number of false positives and focus on shared or convergent signatures. Consecutive $100 \mathrm{~kb}$ DAP windows were grouped and are hereafter referred to as DAP regions.

We further distinguished between two types of DAP: intersamples DAP (IS-DAP), when they are shared between populations in our dataset that contains individuals from the same countries (for example, PEL and Peru, both Peruvian populations); and inter-populations DAP (IP-DAP) when they are shared across populations more distantly related, for instance, European Americans and Caribbean. The populations and the number of haplotypes retained in the two datasets are reported in Supplementary Material, Table S1B. 
Table 1. Admixed American populations under study. $\mathrm{N}$ refers to the number of individuals included in the dataset

\begin{tabular}{|c|c|c|c|}
\hline Population & $\mathrm{N}$ & Country & References \\
\hline $\mathrm{ACB}$ & 68 & Barbados & 1000 Genomes Project \\
\hline AfroAme & 2004 & USA & IlluminaI Control Database \\
\hline Argentina & 133 & Argentina & Lopez Herráez et al. (24) \\
\hline ASW & 55 & SW_USA & 1000 Genomes Project \\
\hline Caribbean & 1112 & Puerto Rico and Dominican Republic & Ghani et al. (31) \\
\hline Chile & 25 & Chile & Lopez Herráez et al. (24) \\
\hline Colombian & 26 & Colombia & Bryc et al. (2) \\
\hline Dominican & 27 & Dominican Republic & Bryc et al. (2) \\
\hline Ecuadorian & 19 & Ecuador & Bryc et al. (2) \\
\hline EuroAme & 1562 & USA & IlluminaI Control Database \\
\hline Maya & 25 & Mexico & Moreno-Estrada et al. (25) \\
\hline Mayas & 21 & Mexico & Li et al. (26) \\
\hline Mexican & 364 & Mexico & Moreno-Estrada et al. (25) \\
\hline PEL & 68 & Peru & 1000 Genomes Project \\
\hline Peru & 85 & Peru & Lopez Herráez et al. (24) \\
\hline Puerto & 26 & Puerto Rico & Bryc et al. (2) \\
\hline PUR & 73 & Puerto Rico & 1000 Genomes Project \\
\hline
\end{tabular}

\section{African DAP}

We identified only a single genomic window with an African DAP present in more than one population (Fig. 1 and Table 2). This IP-DAP window (chr15: 65613 654-65695 283) contains 10 SNPs within two immunoglobulin genes: IGDCC3 and IGDCC4. These SNPs show significant underrepresentation of African ancestry in ACB (Barbados Island) and Dominicans when analysed in the 1090Ind dataset.

\section{European DAPs}

We identified 7 DAP genomic regions with underrepresented European ancestry in the 20Pop dataset, of which 2 are replicated in the 1090Ind dataset; in addition, we found one overrepresented region in the 20Pop dataset. More details about the content of the DAP genomic regions are reported in Table 2.

Strikingly, the major signal was found for IP-DAP region in chromosome 6 extending for a total of 7.8 Mb (chr6:25 312 75533098966) with underrepresented European ancestry in two populations, Caribbean and European Americans (EuroAme). This region contains 373 genes (Supplementary Material, Table S2) including the large locus of the human leukocyte antigen (HLA). Nineteen of the 1079 SNPs typed in our dataset are predicted to have a high functional impact, according to the Combined Annotation Dependent Depletion (CADD) annotation (PHRED-scaled C-score $>20$, Supplementary Material, Table S3, PHRED hereafter). Interestingly, six of the $100 \mathrm{~kb}$ windows included in this region carry high proportions of SNPs with large PHRED values ( $\geq 20 ; 26 \%$ of all SNPs in chr6:31 602 967-31688 217, 10\% in both chr6:26 413 088-26484376 and chr6:29 407 970$29483911,8.3 \%$ in both chr6:28 300 336-28391465 and chr6:32 019 769-32083175 and 7.7\% in chr6:30 823 630-30897022), corresponding to the top $5 \%$ of the distribution estimated on all the genomic windows $(26 \%$ corresponds to $0.0006,10 \%$ to $0.025,8.3 \%$ to 0.039 and $7.7 \%$ to 0.039 ). Overall, the variants in this DAP region belong to 35 different genes, of which 32 are related to HLA.

In this context, a variant ( $r$ 3130618, PHRED $=32$, Supplementary Material, Table S3, Caribbean $Z=-5.5$, EuroAme $Z=-4.8$ ) belonging to GPANK1 gene is associated with MMR (measles, mumps and rubella) vaccination-related febrile seizure (27) and Membranous Glomerulonephritis (28). Inside this region, two SNPs, rs140973961 and rs78331658, not present in our genomewide dataset, have been associated with measles and immune response to the measles vaccine, respectively, in the GenomeWide Association Studies (GWAS) catalog $(29,30)$. To the best of our knowledge, these results might be related to the phenomenon of the 'Columbian Exchange'.

Notably, we observed a neighbouring IP-DAP region (chr6:34 102 061-35495811), distant $\sim 1 \mathrm{Mb}$ from the one reported above, with underrepresented American ancestry for 20Pop and 1090Ind in PEL and Mexican, as shown in Table 2. We registered a similar region, $1.1 \mathrm{Mb}$ (chr6:343 32 179-35 495 811), with an overrepresentation of African ancestry (20Pop dataset) in Caribbean (Min $Z=3.2, \operatorname{Max} Z=3.4$ ) and with values close to the significance threshold in the African Americans (AfroAme, $\operatorname{Min} Z=2.4, \operatorname{Max} Z=2.6$ ).

The important role of HLA in shaping the immunological response in humans may raise the possibility that the new environmental dynamics have triggered adaptive forces that ultimately led to the selection of some African allelic variants.

We identified an additional IP-DAP region with underrepresented European ancestry in chromosome 9. It is identical in both datasets, extending for $157 \mathrm{~kb}$ and containing 44 SNPs (chr9:38 615 175-38771831) located in five genes, shared among African Americans, (AfroAme 20 Pop dataset: Min $Z=-3$, Max $Z=-3.6$; 1090Ind: Min $Z=-3.2$, Max $Z=-3.4$ ), Mexicans (20Pop: $\operatorname{Min} Z=-3.2$, Max $Z=-3.3$; 1090Ind: $\operatorname{Min} Z=-3$, Max $Z=-3.3$ ) and European Americans (20Pop: Min $Z=-5.2$, Max $Z=-6.5$ ). Notably, rs7039377 (chr9:38 675 465; AfroAme: 20Pop $Z=-3$, 1090ind $Z=-$ 2.8; Mexican: 20Pop $Z=-2.99,1090 \mathrm{Ind} Z=-3$; EuroAme: $20 \mathrm{Pop}$ $Z=-5.5$ ) has been associated with obesity-related traits in Hispanic children (31).

Moreover, we observed, in both the analysed datasets (although characterized by different size), a consistent signal of underrepresented European ancestry in chromosome 15 (Fig. 1 and Table 2), shared across seven populations: African Americans, Argentina, Caribbean, European Americans, Mexican, MXL and PUR. Among the others, a SNP (rs2278458, chr15:22 


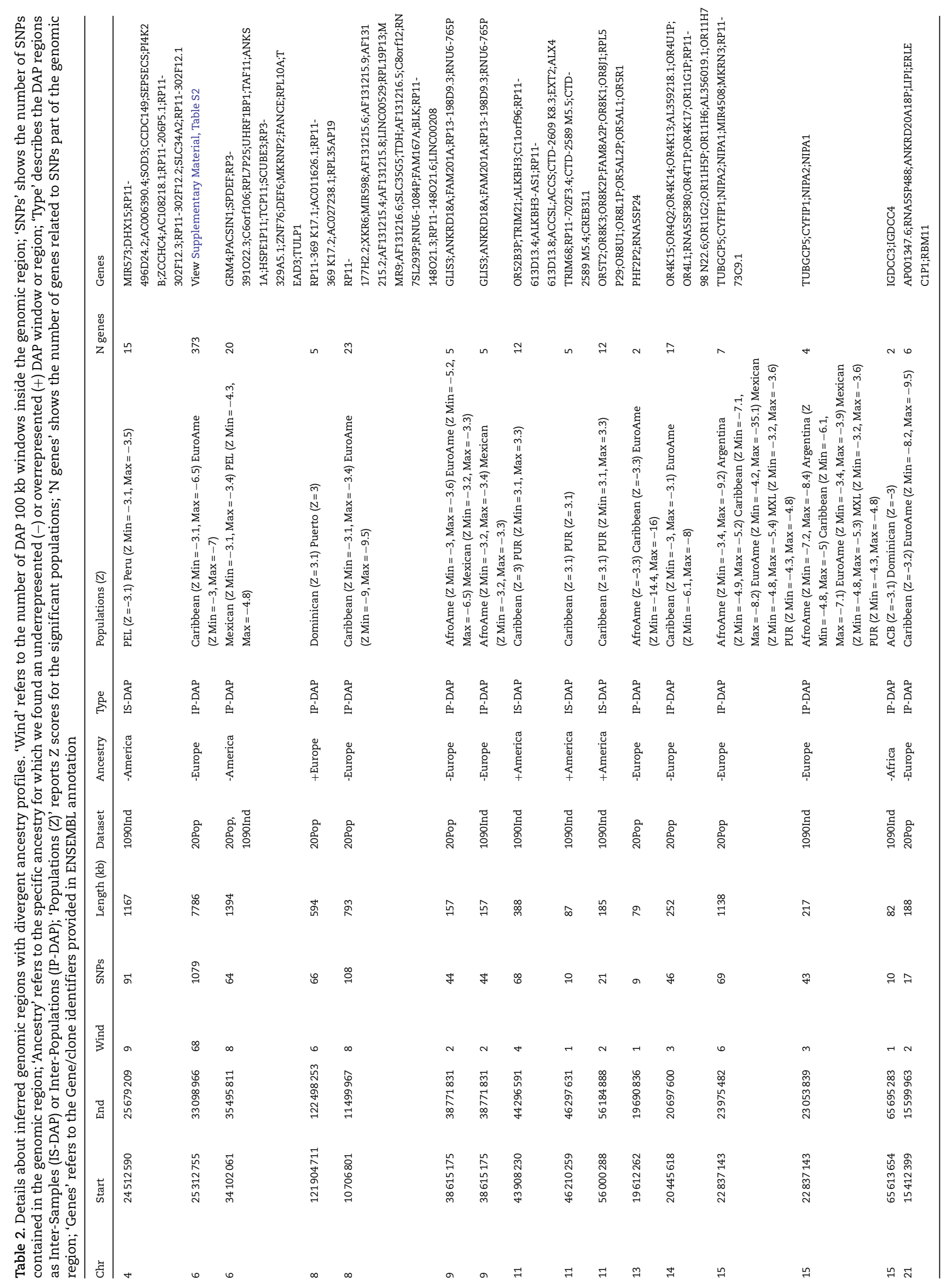


A.

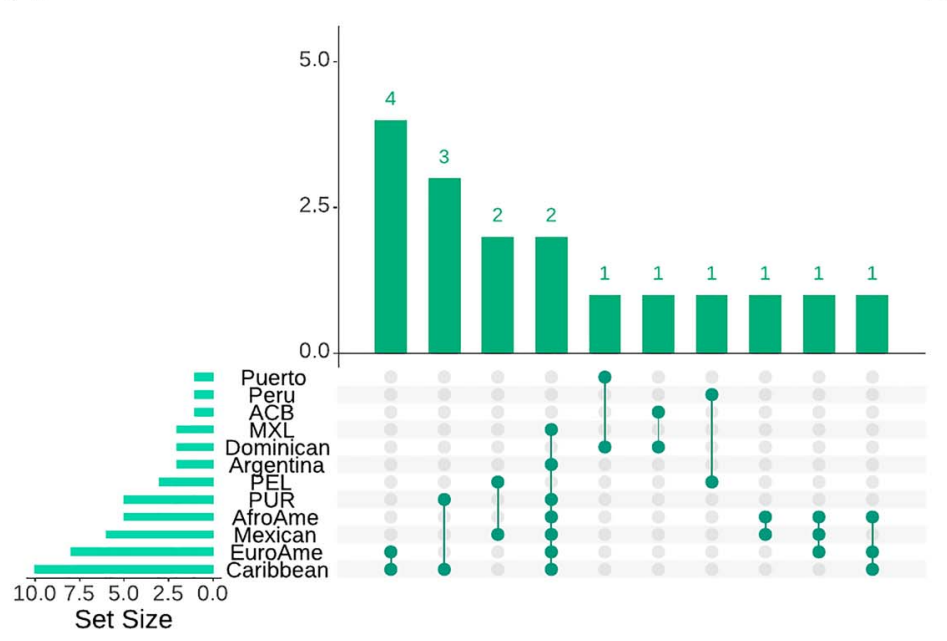

B.

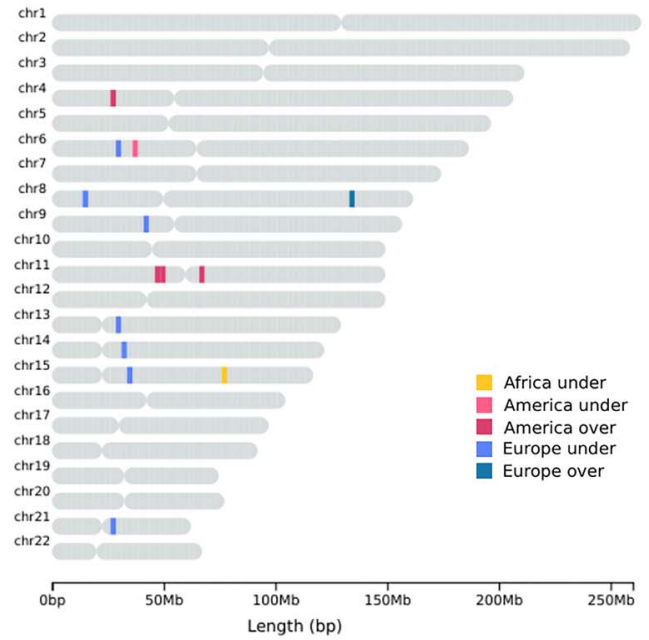

Figure 1. An overview of divergent ancestry profile regions inferred by local ancestry profiles for all ancestries. (A) Population distribution of DAPs. The $\mathrm{x}$ axis shows DAPS in single populations while y axis shows DAP sharing among groups. (B) Genomic location of shared DAPs. Different colours refer to the ancestry and direction of the divergence, as indicated in the legend.

999 857; AfroAme $Z=-8$, Argentina $Z=-5$, Caribbean $Z=-7.4$, EuroAme $Z=-31$, Mexican $Z=-4.8$, PUR $Z=-4.6$ ) in CYFIP1 gene is associated with Triglyceride levels and the response to diuretics in individuals of European and African ancestry (32).

The remaining four IP-DAP regions with significantly lower European ancestry in Caribbean and European American individuals were observed only in the 20Pop dataset and are located in chromosomes: 8, 13 (underrepresented also in African Americans), 14 and 21 (Supplementary Material, Supplementary Text).

Additionally, we identified an IP-DAP region in European ancestry of the 20Pop dataset overrepresented in Dominican and Puerto Ricans (Puerto, Table 2). This region is in chromosome 8 and contains the rs4871180 (chr8:122 259 074; Dominican $Z=3.1$ Puerto $Z=3$ ) that could be associated with Diverticular disease in British individuals (33). The frequency of the risk allele ( $T$ ) of this variant is very high in the analysed Native American source populations, ranging from 0.87 to 0.9 , while it has frequencies of 0.33 and 0.23 in Dominican and Puerto, respectively.

\section{American DAPs}

When the American ancestry profiles were evaluated besides the IP-DAP region of chromosome 6 discussed above, we identified one depleted and three enriched genomic regions in the 1090Ind dataset. Three different IS-DAP regions with observed overrepresentation in Caribbean and Puerto Rico (PUR) were found on chromosome 11 (Fig. 1, Supplementary Material, Supplementary Text and Table 2). They are defined as IS-DAPs because some of the samples included in the Caribbean population are from Puerto Rico (34). The distance between the first two regions is $\sim 2 \mathrm{Mb}$ and could in fact be part of the same selection event. One of these two regions (chr11:46 210 259-46 297 631; $87 \mathrm{~kb}$ ) host a SNP (the rs10437653; Caribbean $Z=2.97$, PUR $Z=2.88$ ) that is associated with birth weight in Europeans (35).

The last region maps on chromosome 4 (chr4:24 512 59025679 209, Table 2) and is underrepresented for American ancestry in two Peruvian populations, PEL and Peru. This is also an IS-DAP region. Interestingly, we identified two SNPs within this region that are associated with diseases/traits: rs12500612 (chr4:24 740 958; PEL Z =-3.1, Peru Z =-3.1) with Major depressive disorder in Europeans (36); and rs1395221 (chr4:24 626 903; PEL $\mathrm{Z}=-3.1$ Peru $\mathrm{Z}=-3.1$ ), with Apolipoprotein A1 and HDL cholesterol levels in European individuals (37). The mean frequency of the risk allele (G) of rs1395221 in the analysed Native American source populations is 0.90 and 0.60 in the European ones, whereas PEL and Peru have a frequency of 0.77 and 0.78 , respectively.

\section{Detecting DAP SNPs in simulated data}

We simulated two datasets with stdpopsim (38) to assess the robustness of our results, as described in the Materials and Methods section. For the first set, 10SIM, we performed 10 independent runs that simulated two admixed American populations (ADM1 and ADM2) to assess how many SNPs with $\mathrm{Z}>|3|$ are replicated in the two simulated populations. For the second set, 100SIM, we conducted 100 independent runs to simulate one admixed population to calculate the corresponding falsepositive rates (Supplementary Material, Fig. S1). For the 10SIM simulated dataset, we did not find any SNP with $\mathrm{Z}>|3|$ in more than one population for any of the 10 independent runs (simulation results are reported in Supplementary Material, Table S4A-B). We obtained single populations signals in some of the admixed simulated populations; however, the amount of significant SNPs in the simulated data is lower than in the real populations, as shown in Supplementary Material, Table S4C. These results suggest that the DAP regions that we detected could not be the result of chance. However, they could be the result of natural selection.

\section{The functional impact of DAP regions in the Americas}

We explored the functional impact measured as SNP deleteriousness (PHRED-scaled C-score) and functional annotation (Annotype), both derived from the CADD annotation, focusing on the multiple population signals described above.

When we compared the distribution of all the PHRED values belonging to windows showing DAPs with the non-divergent ones (non-DAP), European ancestry values were significantly higher in SNPs from DAP windows than those from non-DAP 


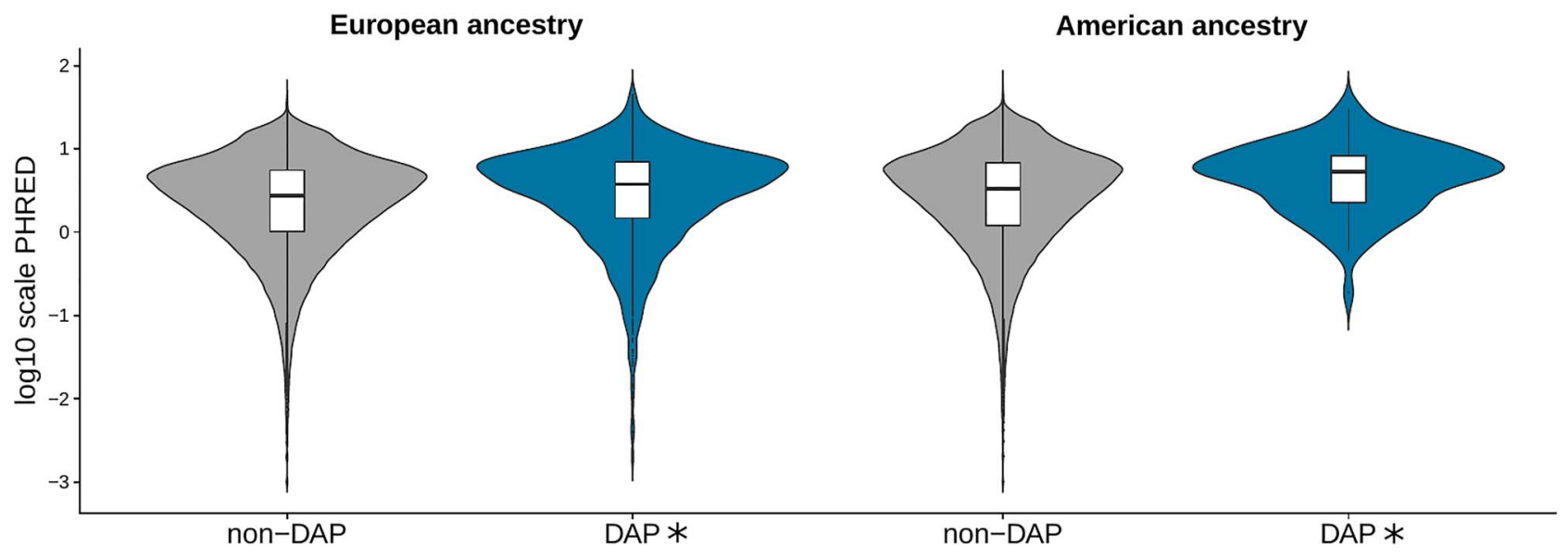

Figure 2. Comparison of the distribution of all the PHRED-scaled C-score values belonging to windows with divergent ancestry profiles (DAPs) with the ones from the non-divergent for European and American ancestries in the 20Pop dataset. The asterisk refers to a statistically significant P-value (Wilcoxon test, Bonferroni corrected alpha $=0.01$ ). The number of analysed windows is reported in Supplementary Material, Table S5A.

A. European ancestry
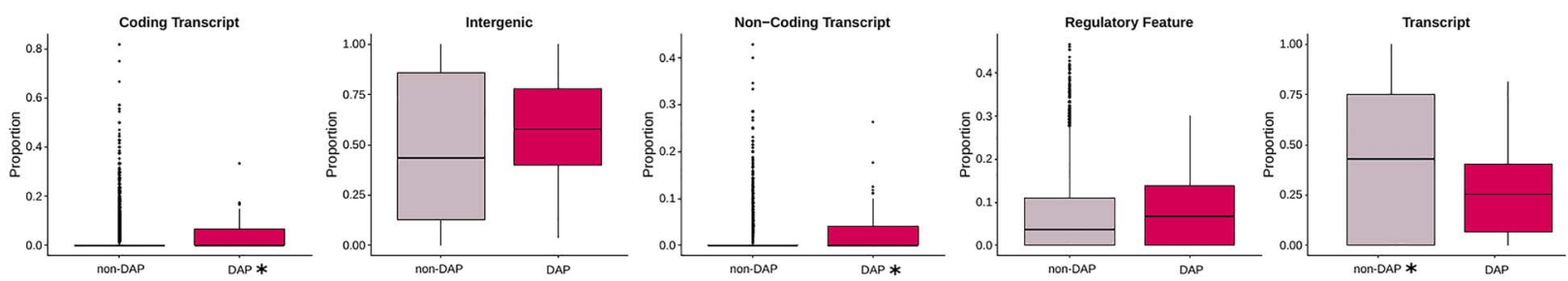

B. American ancestry
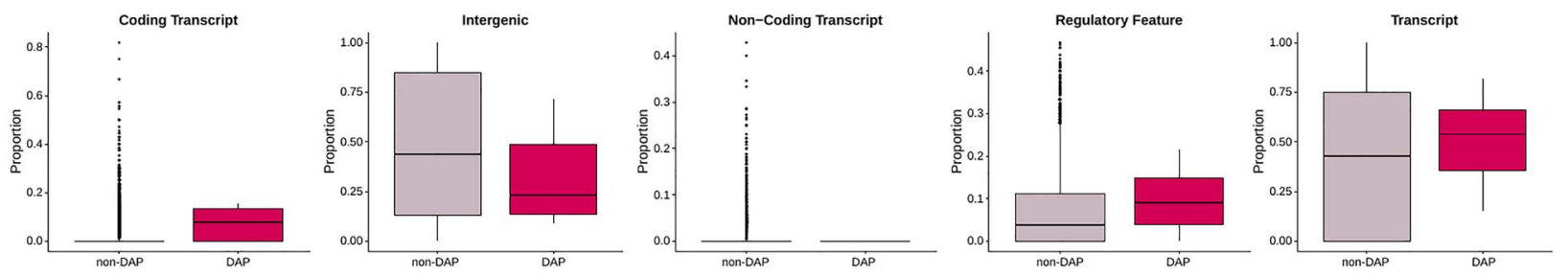

Figure 3. Comparison of the distribution of Annotypes (Coding Transcript, Intergenic, Non-coding Transcript, Regulatory Feature, Transcript) belonging to divergent ancestry profiles (DAPs) windows with the ones from the non-divergent for European (A) and American (B) ancestries in the 20Pop dataset. The asterisk refers to a statistically significant P-value (Wilcoxon test, Bonferroni corrected alpha $=0.002$ ). The number of analysed windows is reported in Supplementary Material, Table S5B.

(Wilcoxon test, $P$-value $=2.8 \mathrm{E}-29)$. The same result was observed for the American ancestry (Wilcoxon test, $P$-value $=0.00014$ ) of the 20Pop dataset (Fig. 2 and Supplementary Material, Table $\mathrm{S} 5 \mathrm{~A})$. No comparison within the 1090Ind dataset showed any significance (Supplementary Material, Fig. S2).

Then, in DAP and non-DAP windows, we analysed the distribution of the proportion of the five types of functional annotation: coding transcript (variants in protein-coding exons), untranslated transcript (variants in untranslated regions (UTRs) and introns), non-coding transcript (mature miRNA and non-coding transcript exon variants), Regulatory Feature and Intergenic. In detail, for each $100 \mathrm{~kb}$ window, we annotated the relative functional composition of all the SNPs and evaluated the proportion of each category (Fig. 3, Supplementary Material, Fig. S3 and Supplementary Material, Table S5B). Every SNP was assigned to at least one Annotype.

We observed that the proportion of 'Coding transcript' ( $P$ value $=8.3 \mathrm{E}-11)$ and 'Non-coding Transcript' $(P$-value $=4.12 \mathrm{E}$ $16)$ is higher in DAP windows than non-DAP in the European ancestry (20Pop Dataset, Wilcoxon test, P-values reported in Supplementary Material, Table S5B, Fig. 3). In contrast, the proportion of 'Untranslated Transcript' is higher in non-DAP SNPs than DAP (European ancestry 20Pop Dataset, Wilcoxon test, $P$-value $=0.00014$, Fig. 3).

Moreover, when we looked at the Native American ancestry, the proportion of 'Coding Transcript' was higher in DAP SNPs than in non-DAP in the 1090Ind dataset (Wilcoxon test, $P$-value $=0.0025$, Supplementary Material, Fig. S3).

\section{Gene set enrichment analysis}

We performed gene set enrichment analysis (GSEA) for the genes encompassing the DAP SNPs to understand if specific health/disease pathways or phenotypes are significantly enriched or depleted in the continental ancestries of multiple populations. We explored five different libraries: Human 2019 Kyoto Encyclopedia of Genes and Genomes (KEGG), Gene Ontology (GO) 2018, GTEx Tissue Sample Gene Expression Profiles down and up and GWAS catalog 2019. 
Interestingly, we found signals exclusively related to underrepresented variants in the European ancestry in the 20Pop dataset from four libraries (GWAS, KEGG, GO and GTEx up) and related to overrepresented variants in the Native American ancestry in the 1090Ind dataset from only one library (KEGG).

In detail, for the European ancestry results, we found 46 significantly overrepresented traits in GWAS Catalog, where the most significant one is related to the Autism spectrum disorder or schizophrenia $(P$-value $=3.9 \mathrm{E}-217)$ term followed by Blood protein levels $(P$-value $=4 \mathrm{E}-84)$ and Ulcerative colitis $(P$-value $=7.7 \mathrm{E}-49)$. Six significant terms are associated with types of cancer: Lung cancer $(P$-value $=1.9 \mathrm{E}-33)$, Squamous cell lung carcinoma $(P$-value $=2.7 \mathrm{E}-31)$, Lung cancer in ever smokers $(P$-value $=1.3 \mathrm{E}$ 29), Small cell lung carcinoma ( $P$-value $=4.1 \mathrm{E}-08)$, Prostate cancer $(P$-value $=0.00011)$ and Cervical cancer $(P$-value $=0.00016)$. Four are connected with Hepatitis $B$, both related to the chronic infection and the response to the vaccine. Then, a group of significant traits is linked to autoimmune diseases such as Sarcoidosis, Psoriasis, Lupus, Behcet's disease, Type I diabetes and Inflammatory Bowel disease.

Eighteen traits are overrepresented when the KEGG library is explored, where the Systemic lupus erythematosus $(P$-value $=2.6 \mathrm{E}$ 46), which is in common with the GWAS library list of terms, has the lowest adjusted P-value followed by Alcoholism $(P$-value $=7.8 \mathrm{E}-27)$ and Viral carcinogenesis $(P$-value $=6 \mathrm{E}$-14). Other terms in common with the GWAS catalogue are Inflammatory Bowel disease (KEGG: $P$-value $=0.00022$, GWAS: $P$-value $=2.7 E-29$ ) and Type I diabetes (KEGG: $P$-value $=1.4 \mathrm{E}-11$, GWAS: $P$-value $=5.9 E-19)$. Finally, significant GO enrichment was found for $\mathrm{MHC}$ class II receptor activity $(P$-value $=2.29 \mathrm{E}-05)$ and GTex lung expression in females of 60-69 years ( $P$-value $=0.00036$ ). The results are presented in Fig. 4, where only the first 10 most significant terms for each annotation library are shown, while in Supplementary Material, Table S6A-D all the results are reported.

When we excluded the gene list related to the chromosome 6 DAP region with underrepresented European ancestry, the GSEA gave no significant results.

For the Native American ancestry, we identified one significant enriched term related to Olfactory Transduction $(P$-value $=0.00047)$ according to the KEGG library (Supplementary Material, Table S6E).

\section{Discussion}

In this study, we evaluated the presence of post-admixture regions with a divergent ancestry proportion on a broad dataset composed of 5828 individuals from 19 recently admixed populations. As previous studies have focused on small sample sizes and a single population, our approach aimed to identify repeated signals at a Pan-American scale, allowing at the same time, the reduction of false-positive rate, and the discovery of selective forces acting at the scale of the entire continent. Furthermore, we considered possible errors by the Local Ancestry algorithm harnessed here, performing the same analysis discarding individuals with extreme values of a given ancestry.

In doing so, despite the potential for African admixtures to enrich the genetic diversity of any recipient human group, among the five populations in the 20Pop dataset and the seven populations in the 1090Ind dataset having a relatively high contribution from Africa, we identified only a single underrepresented and no overrepresented region. Although this scenario is in contrast with surveys identifying DAP regions of African inheritance $(17,18)$, our results might be explained by the high degree of the conservativeness of our approach. Moreover, the recently documented high genetic variation of the African populations contributing to the modern-day American populations, coupled with the relatively low African ancestry proportion in the analysed samples, may have provided differential bases for the subsequent selective forces $(1,39-41)$.

On the other hand, we identified 7 underrepresented regions which consistently showed deviation from the ancestral proportions of the European ancestry, including a $\sim 8 \mathrm{Mb}$ region in chromosome 6 encompassing the MHC II. A neighbouring region was identified as underrepresented also for the American ancestry. Although we failed to identify a replicated signal for African ancestry, it is worth noting that the same HLA has been found overrepresented for the African ancestry in Caribbean, and close to the significant threshold in African American, confirming previous results (13-18).

In this scenario, it might be possible that African variants of the HLA locus might have been the target of selective pressure, also given the new diseases brought by the European populations colonizing the area. Nevertheless, besides this important observation, we did not find consistent and replicated signals for alleles conferring protection for newly introduced pathogens, with the only possible exception of measles. It may be possible that the selection pressure acted independently among different populations, or alternatively, the American populations might already harbour in their genome adaptive variants.

Although a similar signal has been widely replicated, both using genotype arrays and whole-genome sequencing technologies, the high diversity of the region would require an additional confirmation through the analysis of sequence data that uses novel approaches, such as long reads sequencing.

Replicated signals for overrepresented regions that were found in the Americas are consistent with a scenario in which the 15000 years of the peopling of the continents resulted in convergent adaptation.

Our evaluation of the biological impact of post-admixture adaptation in the Americas revealed that irrespective of their direction, SNPs having high functional or phenotypic consequences tend to be 'selected' more often than those with a mild effect. This is also confirmed by the fact that SNPs in UTRs and introns are not a preferential target of natural selection, in contrast to what has been observed for SNPs in coding regions (Fig. 3 and Supplementary Material, Fig. S3).

Lastly, the GSEA performed here revealed that selection acted predominantly on regions associated, among the others, to the onset of autoimmune disease, various protein levels in blood and several different kinds of cancer. The fact that we did not obtain significant results when we removed from the GSEA the underrepresented IP-DAP region in chromosome 6 of European ancestry could be a sign of reduced polygenic enrichment in our data.

Overall, our research suggests that common selective pressure in the Americas had a non-negligible impact on shaping the genetic variation of the two continents, while the Columbian Exchange phenomenon seems to have played just a minor role. Our results also indicate that given the limitations of genetic scan for natural selection algorithms implemented so far, the analysis of multiple population datasets characterized by high sample size will be essential for both the identification and characterization of post admixture adaptation at a more local scale. 


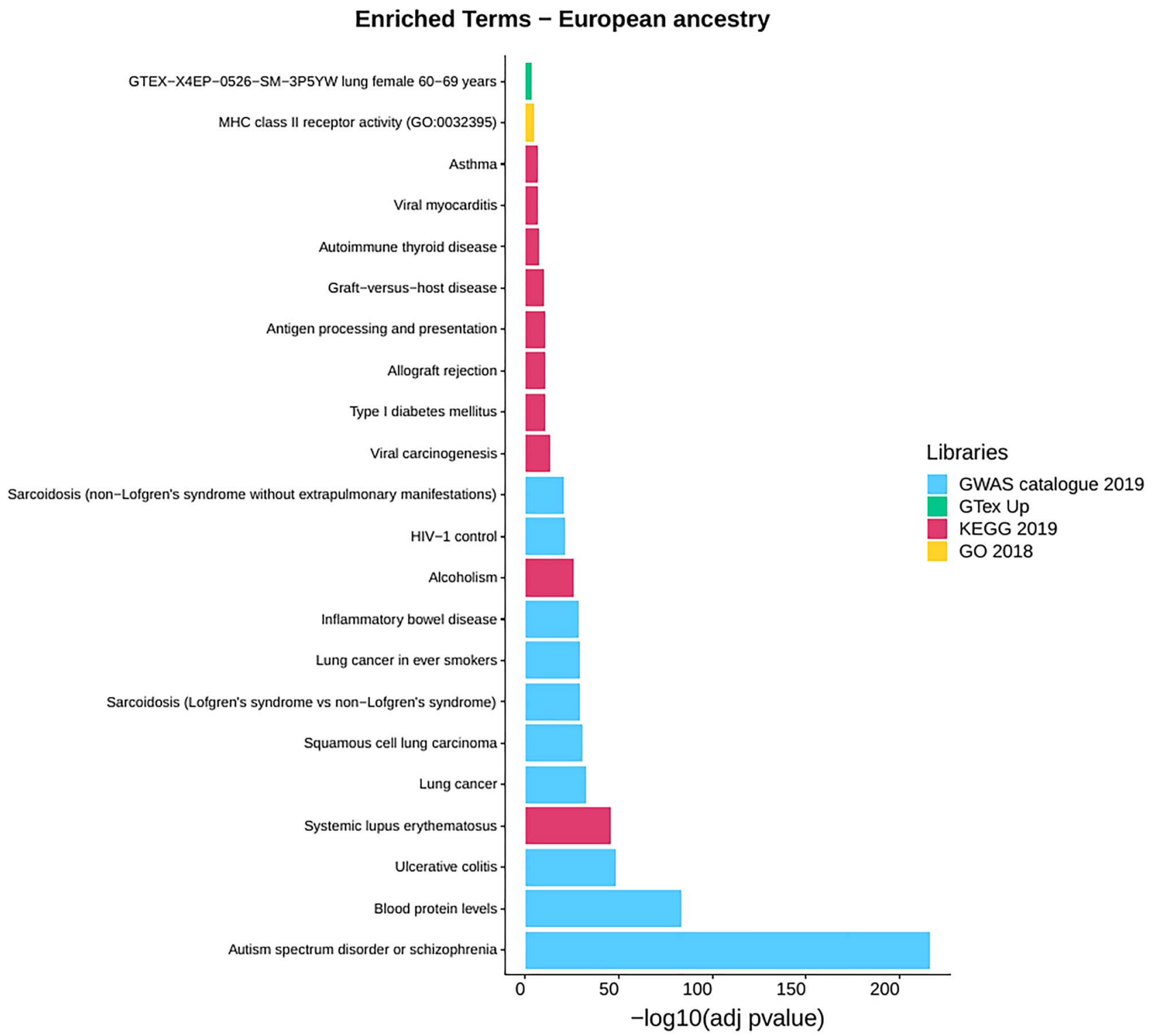

Figure 4. Gene-set enrichment analysis results for the European ancestry of the 20Pop dataset (Supplementary Material, Table S6A-D). Only the first 10 enriched terms for library are shown. Libraries: Genome-wide Association Studies (GWAS) Catalog 2019, GTEx Tissue Sample Gene Expression Profiles up, Human 2019 Kyoto Encyclopedia of Genes and Genomes (KEGG) and Gene Ontology (GO) 2018.

\section{Materials and Methods}

\section{Genome-wide data}

The analysed genome-wide dataset was recovered from Ongaro et al. (1). This dataset was filtered using PLINK ver. 1.9 (42) to include only SNPs and individuals with genotyping success rate $>97 \%$, retaining a total of 251548 autosomal markers. For this study, we used the reference genome version b37. We removed 22295 SNPs belonging to centromeric regions or the first or last $5 \mathrm{Mb}$ of the chromosomes based on the information retrieved from the UCSC browser (https://genome.ucsc.edu/cgi-bin/hgTa bles); after this step, we kept a total of 229253 variants. In the current study, we analysed 6587 individuals, of which 5828 belong to 19 admixed American populations, while the remaining 759 samples are from populations from Africa, Asia, Europe and America. The first set of individuals represents the socalled 'targets', while the second set represents the 'sources'. The details are reported in Supplementary Material, Table S1A-C.

\section{Phasing}

Germline phase was inferred using the Segmented Haplotype Estimation and Imputation tool (ShapeIT2) software (43), using the HapMap37 human genome build 37 recombination map. We used the options-thread 16 and the default value of-effectivesize 15000 as suggested in the manual.

\section{Local ancestry}

We estimated local ancestry assignation for genomic fragments of the target American individuals with RFMIX software (23), using the following reference source populations: Yoruba (YRI), Gambia (GWDwg) and Mozambique for Africa, Chinese Han (CHB) and Japanese (JPT) for Asia, Spanish (IBS), British (GBR) and Tuscany (TSI) for Europe and Tepehuano, Wichi and Karitiana for Native American ancestry (Supplementary Material, Table S1A). 
We used 'PopPhased', '-n 5', and '-forward-backward' options as recommended in the RFMix manual.

Starting from the RFMix output files, we built four PLINK file sets, one for each of the four source ancestries, masking in each one the SNPs assigned to any of the other three ancestries. In details, an allele was assigned as missing in the PLINK file of ancestry A when that allele was not assigned to ancestry A in the 'Viterbi' output file or the probability of belonging to ancestry A (as reported in the 'forward-backward' output) was less than a defined threshold $(<0.9)$. In this and the following analyses, we considered the samples as separated into the two-phased haplotypes.

Given that Asian ancestry was consistently found at low frequency (range:0.2-9.4\%), we decided not to include this ancestry in our search for DAP windows.

At this point, we adopted two different analysis approaches and assembled two datasets (Supplementary Material, Table S1B):

1. 1090Ind dataset: this dataset was assembled considering the ancestral proportions of each individual. In detail, we used the PLINK command-missing to obtain a missing report for each individual; then, using the information from '.imiss' files, we kept those individuals with $>0.1(10 \%)$ and $<0.9(90 \%)$ of F_MISS (representing the missingness of the individual) for each ancestry.

2. 20Pop dataset: this was assembled, taking into consideration the ancestral proportions averaged by populations. In this case, we kept for the analyses only those populations with at least $20 \%$ of a specific ancestry. These proportions were recovered from the global ancestry analysis (SOURCEFIND (4)) reported in Ongaro et al. (1).

\section{SNPs annotation}

We annotated all our SNPs with CADD (Combined Annotation Dependent Depletion, (44)), a tool for scoring the deleteriousness of single-nucleotide variants in the human genome.

\section{Detecting deviation from the expected ancestral proportions}

We estimated the per-SNP average population assignation to detect deviation from the expected ancestral proportions in Local Ancestry Inference.

In doing so, starting from the ancestry-specific PLINK files described above, we initially calculated the population-specific missingness using - missing — family in PLINK 1.9 (42). We then estimated the proportion of Local ancestry assignment as 1missing. The obtained proportions were finally standardized, calculating the Z-score for each SNP. Finally, we partitioned each chromosome into windows of 100000 bp, obtaining 16857 windows, and defined as DAP windows the ones that contained more than five SNPs of which at least one presented a significant $\mathrm{Z}$-score. We decided to set as statistically significant values of $|Z|>3$ (data available at https://doi.org/10.5281/zenodo.4446628). To further reduce the identification of false positives, we focused exclusively on signals replicated in at least two populations. We distinguished between two types of DAP: IS-DAP, when they are shared between populations in our dataset that contains individuals from the same countries (for example, PEL and Peru, both Peruvian populations); and IP-DAP when they are shared across populations more distantly related, for instance, European Americans and Caribbean.
To visualize the results, we used the UpsetR package in $R$ (45) for Fig. 1A, whereas the chromosome map (Fig. 1B) was obtained using the chromoMap package in $\mathrm{R}$.

\section{Validation of our method through simulations}

We performed two separate simulations with stdpopsim (38). Each simulation analysis starts with simulating three source populations (a proxy for African, European and Asian populations), each comprising 50 individuals, then proceed using these populations to create a three-way admixed population. We ran the analyses to simulate the chromosome 1 for 100 individuals per admixed population. The first simulation, named 10SIM, used a slightly modified version of the American population admixture model (AmericanAdmixture_4B11), already implemented in stdpopsim (38). We modified the code to get two independent admixed populations as outputs, ADM1 and ADM2, each comprising 100 individuals. We performed 10 independent runs. The second set, named 100SIM, used the American population admixture model (AmericanAdmixture_4B11) available in stdpopsim (38). We simulated one admixed population with 100 individuals, and we performed 100 independent runs. The subsequent steps were the same for both simulated datasets. The admixed population had an initial size of 30000 and grew at a rate of $5 \%$ per generation, with $1 / 6$ of the population of African ancestry, 1/3 European and 1/2 Asian. We used the HapMapII_GRCh37 genetic map, and we set the parameter ploidy $=2$. To compare the simulation results with the real data, we filtered the simulated SNPs based on the site frequency spectrum of the Caribbean population from our dataset because of the high number of individuals (1112) available for this population, obtaining 19732 SNPs. Then, we applied the same analyses ran on the real data, starting from the local ancestry inference (LAI) using RFMix. From the LAI results, we created three masked datasets by removing the SNPs assigned to the other two ancestries. After running the -missing command with PLINK1.9, we detected those SNPs that diverged from the expected ancestral proportions in the Local Ancestry assignment. We used $Z>|3|$ as the threshold for significance. From the results of 10SIM, we looked at the signals shared between the two simulated populations ADM1 and ADM2, whereas from the results of 100SIM, we estimated the false-positive rate by dividing the number of significant SNPs by the total number of SNPs examined (19732) as reported in Supplementary Material, Table S4B. Supplementary Material, Figure S1 was obtained using the geom_jitter option of the ggplot2 package in R.

\section{PHRED-scaled C-score analysis}

We explored the differences in values of PHRED in the socalled DAP and non-DAP windows. We first extracted from the $100 \mathrm{~kb}$ windows all the SNPs with a corresponding PHRED value retrieved from the CADD annotation. Then, we compared the distribution of all the PHRED values belonging to the DAP windows with the ones from the non-DAP using a paired Wilcoxon test with R. We used a corrected cutoff threshold of 0.01 .

\section{Annotype analysis}

We investigated the differences in the distribution between DAP and non-DAP windows of the five types of functional annotations (Annotype; Coding Transcript, Intergenic, Non-Coding Transcript, Regulatory Feature and Transcript). In CADD annotation, the broad category of transcripts is divided into coding transcript, non-coding transcript and transcript. In details, 'Coding 
Transcript' refers to several types of variants like missense, synonymous, stop-gained, stop-lost, initiator codon, stop-retained, frameshift, inframe insertion, inframe deletion, incomplete terminal codon and protein-altering. The 'Non-Coding Transcript' category contains mature miRNA and non-coding transcript exon variants, while 'Unspecific Transcript' refers to UTRs and introns.

In detail, we extracted all the SNPs from the windows of interest, recovering the corresponding Annotype, then we estimated the Annotype proportions for each window (DAP and non-DAP), and we compared the proportion distributions using a paired Wilcoxon test with R. We used a Bonferroni corrected cutoff threshold of 0.002 .

\section{Gene set enrichment analysis}

GSEA was performed using the GSEAPY enrichr python module on gene lists obtained from the results of the detection of the DAP windows. We focused on the over- and under-represented SNPs from the replicated DAP windows related to the three continental ancestries; then, we extracted the genes assigned to those SNPs by the CADD annotation. In detail, we compiled 7 different gene lists to use as inputs for GSEA. These lists were extracted from multiple population signal results and assembled as follows: (a) 20Pop and 1090Ind European ancestry negative $(Z<-3)$ DAP SNPs, (b) 20Pop European ancestry positive $(Z>3)$ DAP SNPs, (c) 20Pop and 1090Ind Native American ancestry negative DAP SNPs, (d) 1090Ind Native American ancestry positive DAP SNPs and (e) 1090Ind African ancestry negative DAP SNPs.

Only the combinations of datasets and ancestries with at least one DAP window are present.

To identify different aspects of the biological impact of selected DAP windows, we used five diverse libraries:

- Human 2019 KEGG that is a database resource for understanding high-level functions and utilities of the biological system.

- GO Molecular Function 2018.

- Genotype-Tissue Expression (GTEx Tissue Sample Gene Expression Profiles down and up) both for down and up regulated genes.

- GWAS catalog 2019.

We applied a significance cutoff threshold of 0.001, and any adjusted $P$-value below this cutoff was considered significant.

\section{Supplementary Material}

Supplementary Material is available at HMG online.

\section{Acknowledgements}

We thank the people working at the High Performance Computing Center of the University of Tartu for the help and support provided. We thank Marco Rosario Capodiferro for useful discussions. This work was supported by the European Union through the European Regional Development Fund (Project No. 20142020.4.01.16-0030 to LO, MMe, FM; Project No. 2014-2020.4.01.160271 to RF; Project No. 2014-2020.4.01.16-0125 to RF; Project No. 2014-2020.4.01.16-0024 to DM, LP). This work was supported by the Estonian Research Council grant PUT (PRG243) (to RF, MMe, LP). This work was supported by institutional research funding IUT (IUT24-1) of the Estonian Ministry of Education and Research (to TK). This research was supported by the European Union through Horizon 2020 grant no. 810645 (to MMe). This research was supported by the European Union through the Horizon 2020 research and innovation programme under grant no 810645 and through the European Regional Development Fund project no. MOBEC008 to MMO.

Conflict of Interest statement. None declared.

\section{References}

1. Ongaro, L., Scliar, M.O., Flores, R., Raveane, A., Marnetto, D., Sarno, S., Gnecchi-Ruscone, G.A., Alarcón-Riquelme, M.E., Patin, E., Wangkumhang, P. et al. (2019) The genomic impact of European colonization of the Americas. Curr. Biol., 29, 3974-3986.e4.

2. Bryc, K., Durand, E.Y., Macpherson, J.M., Reich, D. and Mountain, J.L. (2015) The genetic ancestry of African Americans, Latinos, and European Americans across the United States. Am. J. Hum. Genet., 96, 37-53.

3. Montinaro, F., Busby, G.B.J., Pascali, V.L., Myers, S., Hellenthal, G. and Capelli, C. (2015) Unravelling the hidden ancestry of American admixed populations. Nat. Commun., 6, 6596.

4. Chacón-Duque, J.-C., Adhikari, K., Fuentes-Guajardo, M., Mendoza-Revilla, J., Acuña-Alonzo, V., Barquera, R., Quinto-Sánchez, M., Gómez-Valdés, J., Everardo Martínez, P., Villamil-Ramírez, H. et al. (2018) Latin Americans show wide-spread Converso ancestry and imprint of local native ancestry on physical appearance. Nat. Commun., 9, 5388.

5. Homburger, J.R., Moreno-Estrada, A., Gignoux, C.R., Nelson, D., Sanchez, E., Ortiz-Tello, P., Pons-Estel, B.A., AcevedoVasquez, E., Miranda, P., Langefeld, C.D. et al. (2015) Genomic insights into the ancestry and demographic history of South America. PLoS Genet., 11, e1005602.

6. Sabeti, P.C., Varilly, P., Fry, B., Lohmueller, J., Hostetter, E., Cotsapas, C., Xie, X., Byrne, E.H., McCarroll, S.A. and Gaudet, R. (2007) Genome-wide detection and characterization of positive selection in human populations. Nature, 449, 913-918.

7. Prohaska, A., Racimo, F., Schork, A.J., Sikora, M., Stern, A.J., Ilardo, M., Allentoft, M.E., Folkersen, L., Buil, A. and MorenoMayar, J.V. (2019) Human disease variation in the light of population genomics. Cell, 177, 115-131.

8. Sabeti, P.C., Reich, D.E., Higgins, J.M., Levine, H.Z.P., Richter, D.J., Schaffner, S.F., Gabriel, S.B., Platko, J.V., Patterson, N.J., McDonald, G.J. et al. (2002) Detecting recent positive selection in the human genome from haplotype structure. Nature, 419 , 832-837.

9. Hejase, H.A., Dukler, N. and Siepel, A. (2020) From summary statistics to gene trees: methods for inferring positive selection. Trends Genet., 36, 243-258.

10. Bryc, K., Auton, A., Nelson, M.R., Oksenberg, J.R., Hauser, S.L., Williams, S., Froment, A., Bodo, J.-M., Wambebe, C., Tishkoff, S.A. et al. (2010) Genome-wide patterns of population structure and admixture in west Africans and African Americans. Proc. Natl. Acad. Sci. U. S. A., 107, 786-791.

11. Jin, W., Xu, S., Wang, H., Yu, Y., Shen, Y., Wu, B. and Jin, L. (2012) Genome-wide detection of natural selection in African Americans pre- and post-admixture. Genome Res., 22, 519-527.

12. Bhatia, G., Tandon, A., Patterson, N., Aldrich, M.C., Ambrosone, C.B., Amos, C., Bandera, E.V., Berndt, S.I., Bernstein, L., Blot, W.J. et al. (2014) Genome-wide scan of 29,141 African Americans finds no evidence of directional selection since admixture. Am. J. Hum. Genet., 95, 437-444.

13. Tang, H., Choudhry, S., Mei, R., Morgan, M., RodriguezCintron, W., Burchard, E.G. and Risch, N.J. (2007) Recent 
genetic selection in the ancestral admixture of Puerto Ricans. Am. J. Hum. Genet., 81, 626-633.

14. Rishishwar, L., Conley, A.B., Wigington, C.H., Wang, L., Valderrama-Aguirre, A. and Jordan, I.K. (2015) Ancestry, admixture and fitness in Colombian genomes. Sci. Rep., 5, 12376.

15. Deng, L., Ruiz-Linares, A., Xu, S. and Wang, S. (2016) Ancestry variation and footprints of natural selection along the genome in Latin American populations. Sci. Rep., 6, 21766.

16. Zhou, Q., Zhao, L. and Guan, Y. (2016) Strong selection at MHC in Mexicans since admixture. PLoS Genet., 12, e1005847.

17. Norris, E.T., Wang, L., Conley, A.B., Rishishwar, L., MariñoRamírez, L., Valderrama-Aguirre, A. and Jordan, I.K. (2018) Genetic ancestry, admixture and health determinants in Latin America. BMC Genomics, 19, 861.

18. Norris, E.T., Rishishwar, L., Chande, A.T., Conley, A.B. and Ye, K. (2020) Admixture-enabled selection for rapid adaptive evolution in the Americas. Genome Biol., 21, 29.

19. Yelmen, B., Mondal, M., Marnetto, D., Pathak, A.K., Montinaro, F., Gallego Romero, I., Kivisild, T., Metspalu, M. and Pagani, L. (2019) Ancestry-specific analyses reveal differential demographic histories and opposite selective pressures in modern south Asian populations. Mol. Biol. Evol., 36, 1628-1642.

20. Nunn, N. and Qian, N. (2010) The Columbian exchange: a history of disease, food, and ideas. J. Econ. Perspect., 24, 163-188.

21. Crosby, A.W. (1987) The Columbian Voyages, the Columbian Exchange, and Their Historians. Essays on Global and Comparative History. In The Columbian Voyages, the Columbian Exchange, and Their Historians. Essays on Global and Comparative History. American Historical Association, Washington, DC.

22. Harper, K.N., Ocampo, P.S., Steiner, B.M., George, R.W., Silverman, M.S., Bolotin, S., Pillay, A., Saunders, N.J. and Armelagos, G.J. (2008) On the origin of the treponematoses: a phylogenetic approach. PLoS Negl. Trop. Dis., 2, e148.

23. Maples, B.K., Gravel, S., Kenny, E.E. and Bustamante, C.D. (2013) RFMix: a discriminative modeling approach for rapid and robust local-ancestry inference. Am. J. Hum. Genet., 93, 278-288.

24. Herráez, D.L., Martínez-Bueno, M., Riba, L., de la Torre, I.G., Sacnún, M., Goñi, M., Berbotto, G.A., Paira, S., Musuruana, J.L., Graf, C.E. et al. (2013) Rheumatoid arthritis in Latin Americans enriched for amerindian ancestry is associated with loci in chromosomes 1, 12, and 13, and the HLA class II region. Arthritis \& Rheumatism, 65, 1457-1467.

25. Moreno-Estrada, A., Gignoux, C.R., Fernández-López, J.C., Zakharia, F., Sikora, M., Contreras, A.V., Acuña-Alonzo, V., Sandoval, K., Eng, C., Romero-Hidalgo, S. et al. (2014) Human genetics. The genetics of Mexico recapitulates Native American substructure and affects biomedical traits. Science, 344, 1280-1285.

26. Li, J.Z., Absher, D.M., Tang, H., Southwick, A.M., Casto, A.M., Ramachandran, S., Cann, H.M., Barsh, G.S., Feldman, M., Cavalli-Sforza, L.L. et al. (2008) Worldwide human relationships inferred from genome-wide patterns of variation. Science, 319, 1100-1104.

27. Feenstra, B., Pasternak, B., Geller, F., Carstensen, L., Wang, T., Huang, F., Eitson, J.L., Hollegaard, M.V., Svanström, H., Vestergaard, M. et al. (2014) Common variants associated with general and MMR vaccine-related febrile seizures. Common variants associated with general and MMR vaccine-related febrile seizures. Nature Genetics (2014), 46, 1274-1282.
28. Stanescu, H.C., Arcos-Burgos, M., Medlar, A., Bockenhauer, D., Kottgen, A., Dragomirescu, L., Voinescu, C., Patel, N., Pearce, K., Hubank, M. et al. (2011) Risk HLA-DQA1 and PLA(2)R1 alleles in idiopathic membranous nephropathy. N. Engl. J. Med., 364, 616-626.

29. Haralambieva, I.H., Ovsyannikova, I.G., Kennedy, R.B., Larrabee, B.R., Zimmermann, M.T., Grill, D.E., Schaid, D.J. and Poland, G.A. (2017) Genome-wide associations of CD46 and IFI44L genetic variants with neutralizing antibody response to measles vaccine. Hum. Genet., 136, 421-435.

30. Tian, C., Hromatka, B.S., Kiefer, A.K., Eriksson, N., Noble, S.M., Tung, J.Y. and Hinds, D.A. (2017) Genome-wide association and HLA region fine-mapping studies identify susceptibility loci for multiple common infections. Nat. Commun., 8, 599.

31. Comuzzie, A.G., Cole, S.A., Laston, S.L., Voruganti, V.S., Haack, K., Gibbs, R.A. and Butte, N.F. (2012) Novel genetic loci identified for the pathophysiology of childhood obesity in the Hispanic population. PLoS One, 7, e51954.

32. de Las Fuentes, L., Sung, Y.J., Sitlani, C.M., Avery, C.L., Bartz, T.M., de Keyser, C., Evans, D.S., Li, X., Musani, S.K., Ruiter, R., Smith, A.V. et al. (2020) Genome-wide meta-analysis of variant-by-diuretic interactions as modulators of lipid traits in persons of European and African ancestry. Pharmacogenomics J., 20, 482-493.

33. Maguire, L.H., Handelman, S.K., Du, X., Chen, Y., Pers, T.H. and Speliotes, E.K. (2018) Genome-wide association analyses identify 39 new susceptibility loci for diverticular disease. Nat. Genet., 50, 1359-1365.

34. Ghani, M., Sato, C., Lee, J.H., Reitz, C., Moreno, D., Mayeux, R., St George-Hyslop, P. and Rogaeva, E. (2013) Evidence of recessive Alzheimer disease loci in a Caribbean Hispanic data set: genome-wide survey of runs of homozygosity. JAMA Neurol., 70, 1261-1267.

35. Warrington, N.M., Beaumont, R.N., Horikoshi, M., Day, F.R., Helgeland, Ø., Laurin, C., Bacelis, J., Peng, S., Hao, K., Feenstra, B. et al. (2019) Maternal and fetal genetic effects on birth weight and their relevance to cardio-metabolic risk factors. Nat. Genet., 51, 804-814.

36. Investigators, G., Investigators, M. and Investigators, S.D. (2013) Common genetic variation and antidepressant efficacy in major depressive disorder: a meta-analysis of three genome-wide pharmacogenetic studies. Am. J. Psychiatry, 170, 207-217.

37. Richardson, T.G., Sanderson, E., Palmer, T.M., Ala-Korpela, M., Ference, B.A., Davey Smith, G. and Holmes, M.V. (2020) Evaluating the relationship between circulating lipoprotein lipids and apolipoproteins with risk of coronary heart disease: a multivariable Mendelian randomization analysis. PLoS Med., 17, e1003062.

38. Adrion, J.R., Cole, C.B., Dukler, N., Galloway, J.G., Gladstein, A.L., Gower, G., Kyriazis, C.C., Ragsdale, A.P., Tsambos, G., Baumdicker, F. et al. (2020) A community-maintained standard library of population genetic models. elife, 9, e54967.

39. Gouveia, M.H., Borda, V., Leal, T.P., Moreira, R.G., Bergen, A.W., Aquino, M.M., Araujo, G.S., Araujo, N.M., Kehdy, F.S.G., Liboredo, R. et al. (2020) Origins, admixture dynamics and homogenization of the African gene pool in the Americas. Mol. Biol. Evol., 37, 1647-1656.

40. Micheletti, S.J., Bryc, K., Ancona Esselmann, S.G., Freyman, W.A., Moreno, M.E., Poznik, G.D., Shastri, A.J., 23 and Me Research Team, Beleza, S. and Mountain, J.L. (2020) Genetic consequences of the transatlantic slave trade in the Americas. Am. J. Hum. Genet., 107, 265-277. 
41. D’Atanasio, E., Trionfetti, F., Bonito, M., Sellitto, D., Coppa, A., Berti, A., Trombetta, B. and Cruciani, F. (2020) Y haplogroup diversity of the Dominican Republic: reconstructing the effect of the European colonization and the trans-Atlantic slave trades. Genome Biol. Evol., 12, 1579-1590.

42. Chang, C.C., Chow, C.C., Tellier, L.C., Vattikuti, S., Purcell, S.M. and Lee, J.J. (2015) Second-generation PLINK: rising to the challenge of larger and richer datasets. Gigascience, 4,7 .
43. Delaneau, O., Marchini, J. and Zagury, J.-F. (2011) A linear complexity phasing method for thousands of genomes. Nat. Methods, 9, 179-181.

44. Rentzsch, P., Witten, D., Cooper, G.M., Shendure, J. and Kircher, M. (2019) CADD: predicting the deleteriousness of variants throughout the human genome. Nucleic Acids Res., 47, D886-D894.

45. Conway, J.R., Lex, A. and Gehlenborg, N. (2017) UpSetR: an R package for the visualization of intersecting sets and their properties. Bioinformatics, 33, 2938-2940. 